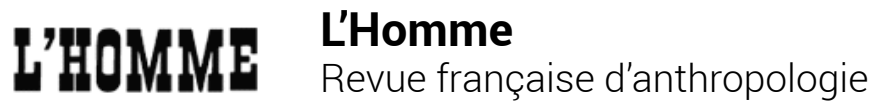

187-188 | 2008

Miroirs transatlantiques

\section{Donna Haraway, Manifeste cyborg et autres essais. Sciences, fictions, féminismes}

\section{Brigitte Steinmann}

\section{(2) OpenEdition \\ 1 Journals}

\section{Édition électronique}

URL : https://journals.openedition.org/lhomme/20592

DOI : 10.4000/lhomme.20592

ISSN : 1953-8103

Éditeur

Éditions de l'EHESS

\section{Édition imprimée}

Date de publication : 3 octobre 2008

Pagination : 479-481

ISBN : 978-2-7132-2186-6

ISSN : 0439-4216

\section{Référence électronique}

Brigitte Steinmann, "Donna Haraway, Manifeste cyborg et autres essais. Sciences, fictions, féminismes », L'Homme [En ligne], 187-188 | 2008, mis en ligne le 16 décembre 2008, consulté le 22 avril 2022. URL : http://journals.openedition.org//homme/20592 ; DOI : https://doi.org/10.4000//homme.20592

Ce document a été généré automatiquement le 22 avril 2022.

(c) École des hautes études en sciences sociales 


\title{
Donna Haraway, Manifeste cyborg et autres essais. Sciences, fictions, féminismes
}

\author{
Brigitte Steinmann
}

\section{RÉFÉRENCE}

Donna HARAWAY, Manifeste cyborg et autres essais. Sciences, fictions, féminismes. Paris, Exils, 2007, $333 \mathrm{p}$.

DONNA HARAWAY, biologiste de formation et «issue d'une famille catholique », ainsi que l'indiquent ses biographes dans la préface de l'ouvrage, a une longue expérience des pratiques scientifiques dans les laboratoires d'étude du vivant: elle a effectué en particulier des recherches sur les primates. Professeur dans un département d'histoire de l'Université de Californie à Santa Cruz, nous pourrions ajouter qu'elle est aussi une philosophe et une anthropologue du politique révolutionnaire, iconoclaste et donc déréificatrice. À travers le Manifeste cyborg, qui réunit l'essentiel de ses essais (Savoirs situés: la question de la science dans le féminisme; Le Patriarcat de Teddy Bear; Ecce Homo, "Ne suis-je pas une femme?"; La Race: donneurs universels dans une culture vampirique; Le Témoin modeste), elle a construit un «mythe politique ironique », le cyborg, «fidèle au féminisme, au socialisme et au matérialisme ", autant de lieux où s'exerce sa critique sociale radicale du "genre »; c'est-à-dire qu'avec l'invention d'un artefact à visée utopiste, inspiré de ses recherches de laboratoire et de terrain, elle veut donner aux femmes et aux hommes un point de vue radicalement nouveau «pour sortir de la vieillerie humaine sexuée et enracinée dans le genre ». Armées du mythe du cyborg, les femmes (et les hommes dans la foulée), devraient enfin parvenir à se «dénaturer ». Il n'est pas certain néanmoins qu'elle destine cette arme à tous les hommes tant il faut un certain éveil et une certaine conscience politiques de la domination générale (particulièrement de la gent féminine), pour mériter le titre de penseur(se) cyborgien(ne). C'est donc par hostilité à toute idée de nature et d'enracinement du 
« genre » dans la nature qu'elle veut inventer en quelque sorte un nouveau genre, le genre cyborgien. Avec la vieille humanité, vient automatiquement l'idée de «reproduction naturelle» ou d'engendrement, ce qui signifie pour les femmes le rattachement à des généalogies patriarcales, enracinées dans des pratiques coloniales de domination et d'asservissement. Ecce Homo nous présente tout un ensemble d'exemples tirés de ses lectures, de ses observations et de ses expériences de vies de femmes à qui n'a jamais été accordé, dans l'Histoire, de position egocentrée (Haraway parle du phallogocentrisme pour les hommes). La femme, lorsqu'elle est de couleur, se voit même dénier le simple droit à la parenté par les mâles. Par exemple, Sojourner Truth, ancienne esclave héroïne et militante abolitionniste de l'ohio, est restée prisonnière comme beaucoup, à son époque, des cadres religieux et messianiques. Donna Haraway, qui dénonce aussi les campagnes religieuses évangélistes de domination des femmes aux États-Unis, interroge ici plutôt les discours coloniaux, racistes et scientifiques, ceux qui se tissent dans les laboratoires et les musées d'histoire naturelle, les discours phallogocentrés autour d'une origine de l'Homme mâle, unique, total, qu'il soit préhistorique ou postmoderne. Impossible de sortir de cette "vision" du mâle sans attaquer les référents dualistes qui la légitiment, les couples nature/culture, esprit/ corps, soi/autre, mâle/femelle, civilisé/primitif, actif/passif, vrai/faux, etc. Il s'agit donc de repenser les rapports politiques et technologiques de sexe et de travail qui placent historiquement les femmes en situation de domination aussi bien matérielle qu'idéelle. S'agit-il pour autant de prôner un monisme? Non, puisque, dit Donna Haraway, «Être un, c'est être autonome, c'est être puissant, c'est être Dieu ; mais être Un est aussi être une illusion, et ainsi être impliqué dans une dialectique apocalyptique avec l'autre. Pourtant, être autre, c'est être multiple, sans bornes précises, effiloché, sans substance. Un, c'est trop peu, mais deux, c'est déjà trop ». Le mythe du cyborg est donc une tentative de désaliénation radicale en termes d'arrachement des femmes au dualisme et de leur recentrement à partir d'un point de vue hors de saisie immédiate, un « point de vue » qui s'inscrit hors-champ, à l'enseigne du mythe. Une telle tentative n'est pas sans poser des difficultés.

Bruno Latour, dont Donna Haraway discute les positions de critique des sciences, s'est lui-même attelé à décrypter les fétiches de la fausse conscience au fait d'attribuer plus ou moins de réalité à des artefacts, selon qu'ils sont produits par l'homme civilisé ou par le sauvage. Il ne s'agit pas de défaire tous les artefacts de femme pour trouver la vraie femme, répondrait Haraway, tout comme le véritable effet hallucinatoire de la croyance résiste à sa démolition et à sa reproduction, avait découvert Bruno Latour : "Comment se passer de l'intériorité et de l'extériorité $»^{1}$ à la fois, se demande-t-il en effet? Malgré son humilité naturelle, que Donna Haraway ne manque pas de louer, et qui lui fait déclarer prudemment "nous sommes tous, comme on dit, dépassés par les événements $"^{2}$, il ne trouvera pas grâce devant la biologiste qui, dans Le Témoin modeste, lui attribue un œil, certes modeste mais néanmoins pervers. B. Latour rétablirait en effet subrepticement le modèle du démiurge à travers les pratiques scientifiques dont il a essayé de démonter les protocoles. Il apparaît que, pour les anthropologues et philosophes français mâles, un passage aux États-Unis ne pouvait avoir qu'un effet bénéfique sur la psyché profonde : entre Bruno Latour et Jacques Derrida, c'est encore le démolitioniste qui s'en tire le mieux... ${ }^{3}$ réflexions anthropologiques et politiques à l'histoire des technologies : «j'ai conscience 
de ce que je dois au cours de l'Histoire dans ma façon de voir les choses: une jeune catholique irlandaise n'aurait jamais obtenu un doctorat de biologie sans l'impact qu'eut Spoutnik sur la politique nationale américaine en matière d'éducation scientifique ». Sa réfutation des thèses des marxistes féministes, qui pourraient lui reprocher malgré tout son idéalisme individualiste occidental ethnocentrique (son choix d'objet mythique est très californien), s'appuie sur une présentation d'elle-même comme "produit» de son terrain, tout autant qu'être conscient-pratique ayant opéré des transmutations personnelles "grâce" au terrain: elle participe des études critiques des cultural studies aux États-Unis par une position d'ethnologue que nous appellerons fictionnaliste, c'est-à-dire libérée de la "science fiction" a-politique ordinaire. Celle-là n'est, en effet, qu'une reproduction technologique des formes humaines de la domination, à la source d'artefacts réifiants que la pensée ordinaire investit de représentations idéologiques coloniales ethno/phallocentriques.

4 Au demeurant, malgré un style cyborgien parfois difficile à suivre, Donna Haraway dénonce l'esclavagisme du travail des femmes américaines (en particulier d'origine africaine et asiatique): maison, marché, lieux du travail salarié, école, clinique ou hôpital et église, autant de lieux où se tissent les réseaux de pouvoir qu'elle veut défaire, « dé-lire » d'un point de vue privilégiant la désidentification des combats des femmes d'avec ceux des travailleurs aliénés en général : il demeure une spécificité de l'aliénation féminine et de l'aliénation du corps des femmes dans l'exploitation capitaliste qu'elle veut déceler et analyser en dehors du champ classique du marxisme. Elle prône une nouvelle identité déterritorialisée, amie de l'artifice. Bienvenue, dit-elle, au bruit, à la pollution et aux fusions illégitimes entre l'animal et la machine. Donna Haraway a dépassé de loin l'anthropomorphisme du Frankenstein de Mary Wollstonecraft : elle lui préfère le Primate fluorescent, l'Homme de Kivu, celui qui se dresse sur la couverture du livre au milieu d'une forêt recomposée comme les familles du même nom. Sa suspicion sur la validité des limites qui séparent les organismes des machines et sur les frontières érigées entre règne animal et règne humain s'avère beaucoup plus révolutionnaire que les positions circonspectes des écologistes de la planète Terre soucieux de «tri sélectif » des déchets-poubelles, qui ne font en réalité qu'aménager de nouvelles frontières hygiénistes. Bienvenue également aux nouvelles "femmes de couleur ", celles qui, armées de biomythographies, de nouveaux concepts comme, par exemple, celui de "Sœur d'Ailleurs" (Audre Lorde ${ }^{4}$ ), permettront de découvrir de nouvelles "délocalisations", trafics sexuels de corps de femmes capitalisés sur les chaînes de montage de systèmes électroniques. Il faut déplacer sans cesse le regard critique afin de l'émanciper de celui de Big Brother. Les thèses antinatures de Donna Haraway rejoignent celles de Valérie Solanas, qui optait pour une "société entièrement automatisée " afin de hâter le processus d'émancipation des femmes. Mais Valérie Solanas n'était ni utopiste ni réificatrice. Après la lecture du Manifeste cyborg, nous conseillons donc vivement aux lectrices de L'Homme de lire ou de relire sans tarder (au moins) la page 81 du manifeste ${ }^{5}$ de Valérie Solanas, pour un érotisme politique total. 


\section{NOTES}

1. Bruno Latour, Petite Réflexion sur le culte moderne des dieux faitiches. Paris, Les Empêcheurs de penser en rond, 1996: 72.

2. Ibid.: 85 .

3. Lui qui a eu la prescience de faire publier de façon posthume L'Animal que donc je suis, Paris, Galilée, 2006.

4. Citée p. 70 (A. Lorde, Sister Outsider, Freedom, Crossing Press, 1984).

5. Valérie Solanas, Scum Manifesto. Association pour tailler les hommes en pièces, Paris, Mille et une nuits, 1998. 